\title{
LES RESSOURCES DE LA RÉSILIENCE POUR NAVIGUER EN EAUX TROUBLES
}

\author{
Author(s) / Auteur(s) : \\ Guy KONINCKX \\ Senior Consultant \\ G.I.R.O.S. et C.I.R.E.R.O. \\ Guy.koninckx@skynet.be
}

\begin{abstract}
Résumé :
L'adversité teinte la trame de nos quotidiens et la résilience fait partie de l'histoire de l'humanité. Bien que le concept de "résilience" trouve son origine en physique son utilisation est déclinée dans bien des disciplines. La définition initiale nous ouvre à ses propriétés. L'utilisation du concept est plus récente, la dimension de rebond est généralement retenue dans des scénarios de transitions. Devenue multiforme, elle s'observe dans les systèmes humains et non humains, a été transposée dans bien des domaines. Etudiée dans les systèmes humains la résilience peut être considérée de plusieurs manières : un processus, une ressource, une capacité que ce soit au niveau individuel, d'une équipe ou d'une organisation. Parmi les acteurs, pointons les tuteurs de résilience, des tisseurs de liens et porteurs de sens. Un acteur particulier le "toxic handler".

La capacité de résilience entre en action dans des contextes déstabilisants. Elle peut se manifester dès avant la survenance d'un choc jusqu'au rebond. C'est ce qui nous permet d'identifier un cycle, d'aborder la temporalité du rebond.

Au niveau du système, des stratégies d'homéostasie et de rupture peuvent être retenues, avant, pendant et après les évènements fragilisants voire traumatisants. De plus, des approches préventives et/ou curatives, précieuses dans des situations complexes, colorent en quelque sorte l'agilité du système humain.

La structure du système constitue un ingrédient de base pour la résilience. Les systèmes opérants disposent de ressources plus importantes que les systèmes non opérants. Certaines conditions sont favorables à l'émergence de la résilience quel que soit le type : résilience de type 1 ou de type 2 . L'enjeu consiste à pratiquer et à développer cette capacité, jamais acquise une fois pour toute, au sein des systèmes en vue d'affronter des scénarios méconnus et complexes et donc leur apprendre à évoluer dans la confusion.
\end{abstract}

Keywords / Mots-clés :

crises, cycle de vie, types de résiliences, rebond, temporalité, acteurs clés, toxic handler

\section{INTRODUCTION}

Aborder la résilience comme une ressource revient à la considérer comme un ensemble de moyens dont peut disposer une personne, une équipe, une organisation ou un collectif. A partir du moment où une organisation navigue en eaux troubles s'amorce un questionnement pour affronter la situation. Dans pareilles circonstances, il n'est pas rare de réaliser que certaines de ses ressources sont ignorées ou sous-estimées. Différents contextes qu'ils soient sociaux, économiques, financiers, environnementaux, climatiques sont à prendre en compte par le système. Des facteurs de résilience peuvent se retrouver dans les parcours individuels et organisationnels. Leurs parcours évoluent dans le temps et sont émaillés d'un éventail de situations tantôt stimulantes tantôt menaçantes. Aux événements inquiétants succèdent des événements rassurants et la confusion relaye la clarté. L'enjeu consiste à explorer la résilience organisationnelle en vue d'identifier les éléments favorables à évoluer en "eaux troubles". Evoluer dans des contextes tissés d'incertitudes relève du quotidien des systèmes humains. Leur défi revient à développer avec agilité les compétences activant les capacités de rebond. 


\section{COMMENT DÉFINIR LA RÉSILIENCE ORGANISATIONNELLE ?}

La résilience considérée comme capacité de rebondir dans des circonstances déstabilisantes remonte à la nuit des temps. Elle fait partie du quotidien. Initialement, le concept a été utilisé en physique avant d'être décliné dans d'autres disciplines. Un glissement sémantique a pu être observé au fil du temps.

Dans le langage courant il se limite à l'art de rebondir. Eviter l'effondrement et retrouver son équilibre revient à privilégier des comportements de type homéostatiques.

Sans conteste, la définition de la physique pour qui la résilience s'exprime en joules par $\mathrm{cm}$ et caractérise la résistance au choc a inspiré de multiples transpositions. La notion de rebond est absente, sauf qu'il peut en être déduit étant que le métal ploie sans rompre.

Alain Richmond (2003) définit la résilience comme étant «la capacité de retomber sur ses pieds, de garder le cap, d'assurer la pérennité d'un organisme ou d'une société, le maintien d'une certaine permanence dans un environnement turbulent.»

Stephane Vanistendael, Jacques Lecomte et Boris Cyrulnik (2000) adoptent une définition transversale : "la résilience est la capacité d'une personne ou d'un groupe à se projeter dans l'avenir en dépit d'événements déstabilisants, de conditions de vie difficiles, de traumatismes parfois sévères".

La résilience concerne non seulement l'individu, elle peut tout aussi bien toucher un groupe voire une collectivité. L'évocation d'événements pointe la dimension contextuelle qui à son tour évolue avec le temps.

Qu'en est-il au niveau organisationnel, au niveau d'un système ?

Gilles Teneau et Guy Koninckx (2016) définissent la résilience organisationnelle en tant que capacité d'un système (constitué d'individus travaillant dans une organisation), confronté à des événements fragilisant et déstabilisant, voire catastrophiques, de mobiliser ses ressources en vue de rester en lien avec sa finalité et se projeter dans son futur, en privilégiant l'un des trois scénarios possibles :

1. Mettre en place les conditions favorables au processus de résilience avant la crise et sans objectif particulier ;

2. Maintenir son activité dans des limites acceptables ;

3. Se redéployer dans des activités d'une autre nature.

De ces définitions retenons quelques éléments clés à savoir les actions de: rebondir, résister, maintenir, rester en lien, se projeter. Voici quelques balises essentielles pour naviguer en eaux troubles.

\section{UNE BOUSSOLE : LE SENS}

Rester en lien avec sa finalité et se projeter dans son futur constituent un élément fondamental pour créer du sens et déjouer les non-sens. Cette démarche contribue en quelque sorte à l'écologie du système. Les eaux troubles des vécus organisationnels apparaîssent dans un environnement pétri de transitions organisationnelles, de restructurations, de fusions ou de crises. Les membres du système se voient ébranlés à des degrés divers. C'est un moment particulièrement propice à l'apparition de différentes formes de démobilisations ; elles fragilisent le système.

Veiller à la fonctionnalité du système demande une vigilance particulière en des temps incertains. Il est utile de la considérer comme un élément nécessaire mais non suffisant. Bénédicte Vidaillet (2003) en souligne l'importance: "Plus les individus sont en mesure de considérer et d'accepter des événements inattendus, plus leur champ de perception devient large et plus ils sont collectivement vigilants et préparés à faire face à l'imprévisibl". Les principales variables de la vigilance collective sont le développement de procédures formelles et informelles de détection et d'anticipation des erreurs, le maintien d'une diversité analytique destinée à élargir les répertoires d'interprétation possibles des informations, une sensibilité accrue aux contextes opérationnels, une capacité à desserrer les liens hiérarchiques pour permettre une fluidité de l'expertise lorsqu'elle est nécessaire et urgente. 
Dans la mesure où les contextes organisationnels sont de plus en plus imprévisibles il appartient à l'organisation de développer des capacités spécifiques au sein du système. Apprendre à évoluer, à s'adapter, à muter sont loin d'être superflus dans des contextes entortillés dans les bouleversements.

Karl Weick (2003) considère la conscience collective comme un processus dans le cadre duquel il y a lieu de stimuler une disposition à agir collectivement avec vigilance. Pour ce faire le système doit être en capacité d'améliorer voire de changer de structures. Un élément clé qu'il retient : pour être un système fiable il faut un système flexible. La conscience collective ainsi que la vigilance font partie du fonctionnement du système et sont assurés par des membres. A titre préventif l'organisation peut prévoir des rôles tels que le parrainage, le mentorat, le tutorat, le coaching dans le but d'accompagner un membre, de fournir du soutien pendant une période déterminée.

\section{LES ACTEURS DE LA RÉSILIENCE : DES PASSEURS}

Nous venons d'évoquer les rôles prévus ou à prévoir par l'organisation formelle. Ils ne sont pas nécessairement les plus signifiants. Une personne jugée "signifiante" par celui qui traverse une situation traumatisante est considérée être "tuteur de résilience". A ce titre ils peuvent être désignés ou l'être de fait, spontanés, choisis, méconnus ou potentiels. Le tuteur désigné n'est pas automatiquement le tuteur effectif car l'élément signifiant est essentiel. De plus, il apparaît que les acteurs de la résilience ne sont pas automatiquement des membres du système. Certains évoluent à l'extérieur du système.

Boris Cyrulnik (2001) souligne l'importance du tuteur de résilience à propos des facteurs de résilience: "En tête vient la rencontre avec une personne signifiante. Parfois une seule a suffi, une institutrice qui en une phrase a redonné l'espoir à l'enfant, un moniteur de sport qui lui a fait comprendre que les relations humaines pouvaient être faciles, un prêtre qui a transfiguré la souffrance en transcendance, un jardinier, un comédien, un écrivain, un quidam ont donné corps à la simple signification : 'il est possible de s'en sortir'. Tout ce qui a permis de renouer le lien social a permis de remanier l'image que le blessé se faisait de lui-même."

Relevons trois actions majeures des tuteurs de résilience auprès des membres du système qui jouent un rôle de "passeur".

- Propager le sens ;

- Procurer les éléments pour traverser les phases de bouleversement ;

- Pourvoir aux interfaces pour assurer la cohérence au sein de l'organisation ;

Quoi qu'il en soit c'est par le lien que la personne émerge, se construit, existe, tisse son identité individuelle et collective. Ce n'est pas dans l'isolement que le processus de reconstruction opère.

Aussi, nous parlons de parcours de la résilience. Celui-ci comprend de multiples processus variables selon les situations, le contexte, l'environnement.

$\mathrm{Au}$ sein d'un système il y a des membres qui contribuent à créer l'émergence des conditions favorables de résilience. En quelque sorte, ils contribuent à l'autopoïèse de l'organisation étant donné qu'ils participent à la production ou à la transformation des autres tout en continuant de participer à sa propre production. Relevons le rôle particulier d'un acteur: celui du toxic handler: porteur de souffrance.

Gilles Teneau (Teneau \& Lemoigne, 2019) distingue trois types de toxic handler appelés générateurs de bienveillance. Il s'agit du porteur de confiance, type 1, du porteur de souffrance, type 2 et du porteur de compassion, type 3 .

Globalement un toxic handler, autrement dit "un générateur de bienveillance". Lorsqu'il est présent dans l'organisation, il peut libérer les énergies individuelles de ses collègues et collaborateurs grâce à la mise en œuvre de ses qualités. En cas de crise, il peut se révéler être un facilitateur de la résilience organisationnelle.»

"Il se voit assigner ce rôle par des collègues qui se tournent vers lui, parce que ce sont des personnes dignes de confiance, calmes, et qui ne porteront pas de jugements." (Teneau \& Koninckx, 2010) 
Il veille à déjouer une détérioration du climat de travail.

Loin d'être gourou il peut être vu comme "ressource rare" du système dans la mesure où naturellement il s'investit pour faire écho aux souffrances engendrées par des situations troublantes. Pour être considérée comme une ressource, elle se doit d'être créatrice de valeur pour l'organisation en valorisant la balance des menaces et des opportunités.

\section{NAVIGUER EN EAUX TROUBLES}

A l'origine d'eaux troubles nous repérons des éléments internes ainsi qu'externes au système, les deux étant étroitement liés. L'évolution des contextes amènent les organisations à se repositionner. Le repositionnement amène son lot de changements, qu'il s'agisse d'adaptations ou de mutations. A titre d'exemples citons quelques exemples suscitant des questionnements au sein du système: l'introduction de nouvelles méthodes de travail, les modifications d'horaires de travail, le travail à domicile, la reconfiguration ou la fusion d'équipes, la réduction de niveaux hiérarchiques, le changement de partenaires sociaux, la délocalisation d'activités, l'arrivée de nouveaux concurrents, la suppression de produits, l'arrivée de nouveaux dirigeants, le transfert d'activité vers une filiale, les changements politiques tels la perspective du brexit, le changement de normes pour l'octroi de subsides, l'évolution de burn-out, les restructurations parfois nécessaires pour l'évolution et la survie de l'organisation sont autant de situations bousculant les équipes et le système. Aussi des comportements de confusion ou de peur impactent les interactions au sein du système. Des relations toxiques voient-elles le jour? Le système demeure-il fonctionnel ou bascule-t-il vers le dysfonctionnel ?

Les eaux troubles charrient des situations confuses. Elles sont de nature à fragiliser ou troubler le système, les sous-systèmes ou les membres au risque de perdre de vue non seulement leurs priorités mais encore leur finalité ainsi que leurs objectifs.

De manière quelque peu paradoxale, interrogeons-nous un instant. N'est-ce pas comme si la situation doit être suffisamment critique pour que les personnes soient preneuses de changement. De menace ne devient-elle pas une opportunité ? Serait-elle source de métamorphose ou de renouvellement ?

\section{LES PARCOURS DU CHOC À LA RÉSILIENCE ORGANISATIONNELLE}

Dans un registre sociologique, Julien Freund (1976) définit la crise en ces termes : "une situation collective caractérisée par des contradictions et ruptures, grosse de tensions et de désaccords, qui rendent les individus et les groupes hésitants sur la ligne de conduite à tenir, parce que les règles et les institutions ordinaires restent en retrait ou sont même parfois déphasées par rapport aux possibilités nouvelles qu'offrent les intérêts et les idées qui surgissent du changement, sans que l'on puisse cependant se prononcer clairement sur la justesse et l'efficacité des voies nouvelle". Au cours de cette phase caractérisée par sa soudaineté et son imprévisibilité, bien qu'il y ait parfois des signes annonciateurs, les repères des individus sont concernés au point d'affecter le sens ainsi que l'efficacité. L'intensité et le caractère inattendu accentuent le risque de la perte de sens.

Différents scénarios amènent l'organisation à naviguer en eaux troubles. Des événements déstabilisants semant confusion et désarroi se trouvent à la source de la survenance des chocs.

Au niveau du fonctionnement du système on peut observer des manifestations de perturbations sans toutefois être apparentes : il s'agit de "signaux faibles". Au fil du temps elles prennent de l'ampleur et une prise de conscience de la menace se précise. Une phase de gestation précède la crise et survient le choc. Les équipes se referment ou se murent au point de dénier ce qui se profile. Soit les membres du système perdent leurs repères et une phase de déconstruction prend le pas, soit ils se mobilisent pour affronter la situation de crise et d'autres perspectives voient le jour. Des similitudes avec la courbe du deuil d'Elisabeth Kubler Ross, reprises ci-dessous sont éclairantes. Elle nous permet d'appréhender l'itinéraire des systèmes confrontés à naviguer en eaux troubles, nous éclaire sur les scénarios en situation de déconstruction de sens afin de limiter la probabilité d'effondrement. Elle munit préventivement les membres de ressources pour affronter les événements déstabilisants, les 
turbulences et les chocs qui tissent le quotidien des organisations humaines : systèmes, sous-systèmes et membres.

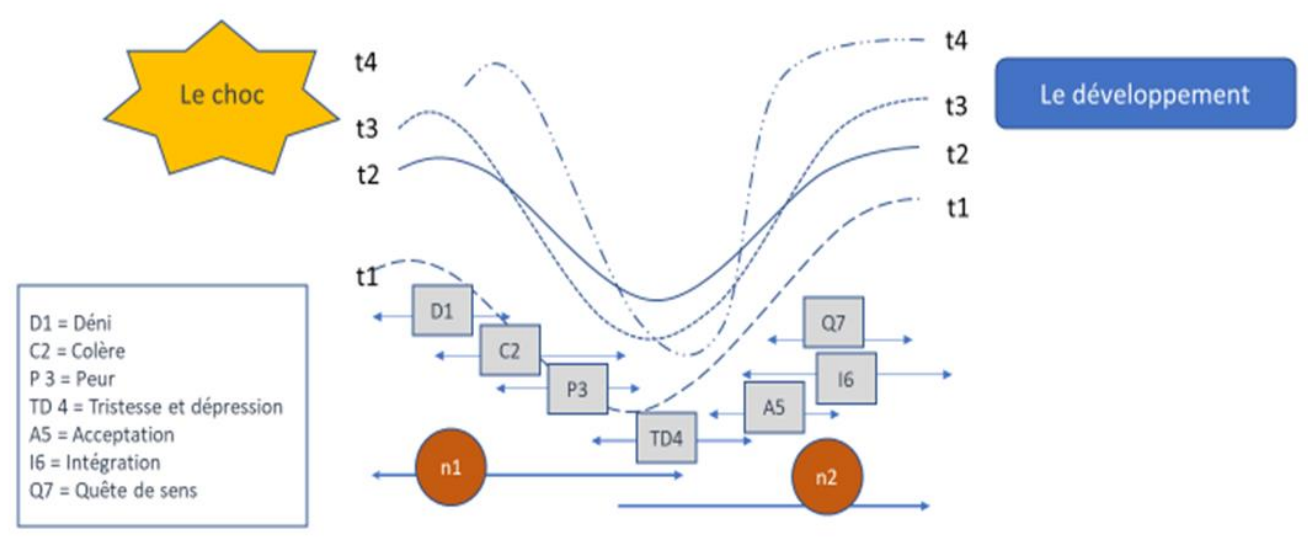

Figure 1 Les parcours du choc à la résilience organisationnelle

Différents parcours de résilience organisationnelle sont représentés.

Les courbes $\mathrm{t} 1, \mathrm{t} 2, \mathrm{t} 3$, $\mathrm{t} 4$ représentent différents parcours de sous-systèmes ou de membres au sein d'un système confronté à un choc, à un contexte déstabilisant.

Le fléchissement des courbes s'inscrit dans le temps et leur amplitude renseigne sur la durée du parcours. Des trajectoires de durées différentes sont constatées ; t $1, \ldots$ à $t 4$ représentent le parcours d'un sous-système voire d'un membre. Force est de constater que sous l'effet d'un même événement, différents comportements sont observés : les sous-systèmes (t1 à t4) sont concernés à des moments différents.

A titre d'exemple 4 se trouve non seulement impacté après $t 1$ mais sa traversée des différentes étapes s'effectue à une autre allure que $\mathrm{t} 1$; la phase de reconstruction est plus courte et rapide que les parcours de $\mathrm{t} 1, \mathrm{t} 2$ et $\mathrm{t} 3$.

Quelle que soit l'allure des courbes nous identifions deux périodes clés. Au temps de déconstruction (n1) caractérisé par le déni, la colère, la peur, voire la peur et la dépression succède un temps de reconstruction (n2) caractérisé par l'acceptation, l'intégration, la quête de sens et le redéploiement des équipes.

\section{LA TEMPORALITÉ DU REBOND}

La résilience, processus s'inscrit dans le temps. On peut parler d'un avant, d'un pendant et d'un après les éléments déstabilisants, les signes précurseurs, les manifestations de la crise et la crise en tant que telle sans perdre de vue l'après crise un temps de consolidation.

\section{LES FORMES DE RÉSILIENCE ORGANISATIONNELLE}

Reprenons notre définition de la résilience organisationnelle vue comme la capacité d'un système (constitué d'individus travaillant dans une organisation), confronté à des événements fragilisant et déstabilisant, voire catastrophiques, de mobiliser ses ressources en vue de rester en lien avec sa finalité et se projeter dans son futur. Elle précède le changement. Trois formes sont à retenir : l'effondrement, la résilience de type 1 et la résilience de type 2 (Freund, 1976). 


\section{L'effondrement ou forme 1}

Dans la mesure où la phase de perte de repères et de sens s'implante, l'absence de rebond se profile. L'énergie fait défaut et le déficit de ressources se solde progressivement par une rupture.

\section{La résilience de type 1 - Forme 2}

C'est l'expression des aptitudes à mobiliser les énergies nécessaires pour retrouver un état d'équilibre antérieur au choc et ce dans des limites acceptables. La perspective temporelle est constituée du présent et du passé, malgré l'absence de préparation avant la crise. C'est vivre avec le choc tout en évoluant dans des limites acceptables pour l'organisation.

\section{La résilience de type 2 - Forme 3}

La résilience de type 2 exprime les aptitudes à mobiliser les énergies nécessaires pour passer la crise et trouver un nouvel équilibre en rupture avec la situation antérieure. Une préparation des conditions de résilience mises en place pendant la phase qui précède les événements traumatiques facilite la traversée. Dans un premier temps elle vit avec le choc dans des limites acceptables avant de réaliser une bifurcation ou une rupture avec le passé. La résilience de type 2 s'inscrit dans le présent et l'avenir.

La résilience est l'aptitude à libérer l'énergie nécessaire pour amorcer le processus de changement 1 ou de changement 2 identifiés par l'école de Paolo Alto (Watzlavick, Weakland \& Fisch, 1975). En ce sens, la résilience constitue une condition nécessaire aux processus de changement.

\section{DES CONDITIONS FAVORABLES À L'ÉMERGENCE DE LA RÉSILIENCE ORGANISATIONNELLE}

Sans être exhaustif nous reprenons quelques points d'attention favorables à l'émergence de la résilience. La plupart des comportements répertoriés découlent des caractéristiques des systèmes fonctionnels du modèle du "giroscope" développé par Andrée Piecq (2011). Le point commun entre ces comportements c'est l'action et ce qui les caractérise c'est la dynamique qu'ils initient au sein d'un système.

- Préciser les orientations générales, les buts et les objectifs ;

- Définir et clarifier les missions, rôles et fonctions ;

- Expliciter et clarifier le sens et le non-sens ;

- Expliciter les règles de fonctionnement et les actualiser ;

- Activer la circulation de l'information interne et externe ;

- Pratiquer et stimuler l'usage des rétroactions.;

- Apprendre à fonctionner avec des séquences de stabilité et de changements ;

- Prendre appui sur les réussites ;

- Accepter l'expression d'émotions ;

- Développer des interactions basées sur l'intelligence émotionnelle ;

- Reconnaître les différences des membres comme ressource ;

- Prévoir des temps et des lieux de parole ;

- Valoriser l'autonomisation des personnes et des départements ;

\section{CONCLUSIONS ET PERSPECTIVES}

Développer la vigilance collective constitue une action à entreprendre, à stimuler et à entretenir pour faire face aux turbulences internes et externes du système. 
L'évaluation du fonctionnement du système donne accès à des informations précieuses. Elle met en évidence non seulement les fragilités du système mais informe sur les cohérence et incohérences présentes dans l'organisation. Indirectement elle permet de pointer les gaspillages d'énergie.

La résilience ne se prépare pas dans l'isolement. Elle se construit par et dans les liens. L'activation des liens ressource les équipes et active les interactions.

Des relations écologiques constituent une base pour le déploiement de la résilience organisationnelle au sein du système. De manière imagée parlons de relations 'anthropogènes' plutôt qu' anthropophage'.

Les contextes du système évoluant, il en résulte la nécessité d'actualiser régulièrement les capacités de rebond du système.

Le toxic handler, porteur de souffrance, générateur de bienveillance constitue une ressource rare pour le système.

L'agilité du système humain est une ressource pour évoluer dans un environnement tissé d'incertitudes.

La résilience est une ressource pour naviguer en eaux troubles, en temps incertains et méconnus.

\section{REFERENCES}

CYRULNICK, B. (2001). Les vilains petits canards. Paris, Odile Jacob p 261

FREUND, J. (1976). Observations sur la dynamique prolégomènes de la crise du conflit. Communications

PIECQ, A. (2011). De la pensée systémique à la pratique de l'organisation. Paris, L'Harmattan.

RICHMOND, C. (2003). La Résilience économique. Paris, Ed. d'Organisation.

TENEAU, G \& KONINCKX, G. (2016). « Comprendre la résilience organisationnelle ». Odile Jacob, IONESCU, S. Résiliences Ressemblance dans la diversité.

TENEAU, G. \& KONINCKX, G. (2010). Résilience organisationnelle - Rebondir face aux turbulences. Bruxelles, Ed. De Boeck.

TENEAU, G. \& LEMOINE, G. ( 2019). Toxic Handlers Les générateurs de bienveillance. Paris, Odile Jacob.

VIDAILLET, B. (2003). Le sens de l'action. Paris, Ed. Vuibert.

WATZLAVICK, P., WEAKLAND, J., FISCH, R. (1975). Changements paradoxes et psychothérapies. Paris, Éd. du Seuil.

WEICK, K. (2003). Le sens de l'action. Paris, Ed. Vuiber. 
